\title{
Lidil
}

Revue de linguistique et de didactique des langues

45 | 2012

Pratiques de formation à la lecture-écriture des adultes en parcours d'insertion

\section{Discours des apprenants sur les effets de la formation à l'écrit}

Marie-Hélène Lachaud et Djamal Rehaili

\section{OpenEdition}

\section{Journals}

Édition électronique

URL : http://journals.openedition.org/lidil/3213

DOI : $10.4000 /$ lidil.3213

ISSN : 1960-6052

Éditeur

UGA Éditions/Université Grenoble Alpes

Édition imprimée

Date de publication : 15 mai 2012

Pagination : 151-162

ISBN : 978-2-84310-226-4

ISSN : $1146-6480$

Référence électronique

Marie-Hélène Lachaud et Djamal Rehaili, « Discours des apprenants sur les effets de la formation à l'écrit », Lidil [En ligne], 45 | 2012, mis en ligne le 15 novembre 2013, consulté le 21 décembre 2020. URL : http://journals.openedition.org/lidil/3213 ; DOI : https://doi.org/10.4000/lidil.3213 


\title{
Discours des apprenants sur les effets de la formation à l'écrit
}

\author{
Marie-Hélène Lachaud* et Djamal Rehaili**
}

\begin{abstract}
RÉSUMÉ
Cet article porte sur une étude qualitative réalisée auprès d'adultes en insertion professionnelle, inscrits dans un parcours de formation « compétences clés ». L'objectif principal est d'appréhender et de décrire la perception qu'ont les apprenants de leur formation, à travers leurs discours et dans la perspective d'améliorer les dispositifs. Ainsi, notre enquête place les apprenants en situation réflexive, à partir de leurs représentations de la formation à l'écrit. Si les résultats montrent des attentes vis à vis des pratiques et des modalités formatives, ils présentent également des interrogations de la part des apprenants.
\end{abstract}

\begin{abstract}
This article deals with a qualitative study carried out with adults entering the labour market, enrolled in the training course entitled "key skills". The main goal is to comprehend and describe adult learners perception about their training through their views, which will help improving the training planning. Thus, our investigation looks at adult learners put into a reflexive situation, taking into account their points of view expressed in writing regarding the training. If the results show the expectations about the training practices and conditions, they also present questions which were raised by the adult learners.
\end{abstract}

La recherche présentée dans cet article est centrée sur les apprentissages des adultes inscrits dans des parcours intitulés « compétences clés » et concerne plus particulièrement des employés en situation d'insertion professionnelle.

\footnotetext{
* Formatrice-consultante ; laboratoire LIDILEM, Université de Grenoble.

** Laboratoire LIDILEM, Université de Grenoble.
} 
Notre étude est issue d'une réflexion menée au sein de Graffic $^{1}$ et fait l'objet d'une mission qui s'inscrit dans le projet régional Rhône-Alpes «Université Solidaire et Citoyenne ». Un des objectifs de notre mission au sein de ce projet est d'appréhender la manière dont les apprenants perçoivent leur formation, ce qu'ils en pensent et ce qu'ils font de leurs apprentissages.

Nous présentons les premiers résultats de notre étude, après avoir délimité le cadre théorique et exposé la méthodologie de la recherche.

\section{Littéracie, représentations et place de l'apprenant dans la formation à l'écrit}

\section{Apprentissage de l'écrit et représentations}

Les travaux des chercheurs réalisés au cours de ces dernières décennies montrent la complexité liée à l'usage de l'écrit et à son apprentissage. Le terme de littéracie prend en considération cette complexité à travers des éléments comme la compréhension, le raisonnement ou les représentations, en plus des activités mentales générées par la lecture et l'écriture (Jaffré, 2004). Cette conception du terme littéracie procure à notre recherche la possibilité d'interroger l'apprentissage de l'écrit sous l'angle des représentations. Envisager l'écrit dans les formations de cette manière revient à considérer les notions de compétence scripturale et de rapport à l'écrit comme deux éléments essentiels (Dabène, 1992; Barré-de Miniac, 2000; Besse et al., 2003 ; Leclercq, 1999). Les représentations constituent un des éléments de la compétence scripturale. En effet, comme le montrent les travaux de recherche, les actes de lectureécriture mobilisent des savoirs et des savoir-faire spécifiques qui sont associés à des représentations et aux motivations personnelles. Ainsi, lorsque l'on s'intéresse à la formation à l'écrit des adultes en parcours d'insertion sociale et professionnelle, les ressentis exprimés ou encore les opinions, sont des éléments importants à prendre en considération.

\section{L'apprenant, principal acteur de sa formation}

L'évolution des pratiques formatives place l'apprenant au centre de l'apprentissage. En effet, d'un point de vue historique, nous sommes passés

1. Groupe de recherche action formation français, insertion, compétences. 
de la « société éducative » de Joffre Dumazedier (1978), à la « société pédagogique » de Jacky Beillerot (1982) pour arriver à la « société dite cognitive » des années 2000. Le paradigme de «l'apprendre », qui comporte le sujet apprenant comme responsable de son apprentissage, s'avère incontournable. Constat majeur en didactique puisque l'apprenant se trouve «non par décret mais par nature » au centre du dispositif de l'apprentissage (Penloup, 1995 : 21). Dans cette même perspective la démarche du Groupe régional de lutte contre l'illettrisme (GRLI, 2003) associe l'apprenant à l'évaluation de ses compétences langagières écrites et l'accompagne dans une attitude réflexive sur ses apprentissages pour une « identification positive des compétences en acte » (p. 14). L'apprenant devient actif dans le processus d'apprentissage. Ainsi, il construit « du sens sur sa propre démarche de formation » (p. 15). Cette conception rejoint celle d'Anne Vinérier (2004) qui, dans le sillage des travaux de Paulo Freire, place l'apprenant au centre de sa formation. Dans la démarche formative " des chemins de savoirs ", reliée à la notion de « conscientisation », ce dernier est associé, dès son entrée en formation, au repérage des savoirs existants (Vinérier, 2004 : 11). Il est conduit à déterminer avec l'aide du formateur, les savoirs qu'il souhaite développer. Notre contribution s'inscrit dans ces mêmes perspectives émancipatrices de l'apprenant, acteur de sa formation.

De plus, dans le cadre de l'évaluation formative, « en sollicitant son avis sur la formation », l'apprenant est invité à se placer dans une posture réflexive par rapport aux tâches de lecture-écriture mais aussi par rapport au dispositif de formation (Leclercq, 1999 : 164). Dans cet article, nous nous intéressons aux propos recueillis dans cette posture et donc aux opinions relatives aux objets d'apprentissage relatifs à l'écrit. Il ne s'agit donc pas ici d'appréhender la dimension cognitive de l'évaluation qui repose sur l'usage des tests. Ainsi, dans le processus d'évaluation, nous retiendrons la dimension psychosociale qui met l'accent sur les représentations de l'écrit et de son apprentissage. Nous considérons les représentations comme une partie du processus de l'auto-évaluation. Ceci, dans la mesure où les discours des apprenants c'est-à-dire leurs opinions, leurs ressentis et leurs perceptions seraient inclus dans ce processus. Notons que ces discours pourraient avoir un impact sur la pratique didactique des formateurs et sur l'élaboration des dispositifs de formation. 


\section{Choix méthodologiques}

\section{Une recherche descriptive}

La démarche méthodologique de notre recherche descriptive est centrée sur l'apprenant. Il s'agit d'une enquête qualitative. Nous avons interviewé et observé douze témoins inscrits dans un module de formation aux « compétences clés ». Cette formation a eu lieu de septembre à décembre 2010, à raison d'une journée par semaine. Les apprenants, que nous avons rencontrés un mois après le démarrage, ont suivi leur formation pendant un peu plus de 80 heures, au total.

Dans le cadre de cet article, nous présentons les premiers résultats pour trois témoins représentatifs de notre échantillon, en ce qui concerne l'âge, l'appartenance culturelle, les besoins de formation et les attentes. Notons que ces critères représentent l'hétérogénéité qui caractérise habituellement les groupes de formation à l'écrit des adultes.

\section{Explicitation et entretiens semi-directifs}

Dans le sillage des recherches en sciences sociales et pour faciliter la mise en confiance de nos témoins, nous avons procédé à une première phase de prise de contact (Blanchet, 2005). Nous avons ensuite débuté notre enquête par des observations de séances. Dans la mesure où notre objectif était de recueillir des opinions sur la formation et sur les pratiques formatives, les échanges qui ont eu lieu entre les chercheurs et les apprenants, pendant ces observations, étaient basés sur la méthodologie de l'entretien d'explicitation (Vermersch, 2006). Ces entretiens, parfois informels, portaient sur les objets d'apprentissage tels que les exercices ou les supports utilisés par les apprenants. Nous avons complété ce recueil de données par des entretiens individuels, semi-directifs, réalisés en dehors des séances de formation. Nous avons enregistré et transcrit les propos recueillis au moment des observations et des entretiens individuels.

Les résultats que nous présentons dans cet article sont donc issus des entretiens semi-directifs ainsi que des échanges informels recueillis lors des observations de séance. Soulignons l'intérêt porté par les témoins à la situation d'entretien, ainsi que leur volubilité lors des échanges. 


\section{Présentation des témoins et attentes de formation}

Bachir : la « remise à niveau ${ }^{2}$ », une étape indispensable à l'aboutissement de son projet professionnel.

Des trois témoins, Bachir est le plus jeune, il a 33 ans. Il vit avec sa femme et ses quatre enfants en bas âge. D'origine algérienne, né et scolarisé en France, Bachir a quitté l'école en troisième année de collège avec un certificat de formation générale. Il a ensuite repris ses études et a obtenu un CAP en boulangerie. L'objectif qu'il vise à travers cette formation est une « remise à niveau » notamment en orthographe et en mathématiques. Deux compétences qui, à son avis, lui permettront de réaliser son projet professionnel : créer son activité de restauration rapide.

Frédérica : être autonome dans les usages sociaux de l'écrit.

Née au Portugal, Frédérica a 47 ans. Sa scolarité a été interrompue en fin de cycle élémentaire. Lorsqu'elle est arrivée en France, en 2006, elle ne parlait ni ne comprenait le français. Au début de la formation, elle souhaite travailler l'écrit et savoir compléter les documents administratifs. Frédérica veut également aider son fils qui est collégien. Comme Bachir, elle aimerait créer sa propre activité professionnelle : une association d'aide à domicile.

Jean-Louis : avant tout « faire le point » sur ses connaissances.

À 51 ans, Jean-Louis est le plus âgé des trois témoins. Il est né et a suivi sa scolarité en France, jusqu'à l'âge de 13 ans. Il occupe un emploi d'ouvrier forestier, dans le cadre d'une insertion professionnelle. JeanLouis était charpentier. C'est la première fois qu'il effectue une formation, il dit qu'il veut «faire le point » sur ses connaissances en français.

\section{Résultats : perception et appréciation des effets de la formation à l'écrit}

Nous présentons ci-dessous les résultats à partir de trois thèmes d'analyse récurrents. Il s'agit de l'opinion des apprenants à propos de leur formation à l'écrit. Ensuite, nous nous focalisons sur la manière dont ils perçoivent l'organisation des séances ainsi que les contenus relatifs aux apprentissages de l'écrit. Enfin, nous resserrons notre analyse autour de

2. Les propos des témoins sont transcrits entre guillemets. 
l'évocation des apports de la formation et des éventuels changements par rapport aux pratiques de lecture et d'écriture, intervenus dans le contexte professionnel, social ou familial des apprenants. À noter que nous complétons la présentation de leurs points de vue par nos propres commentaires. Ceci, dans la perspective d'ouvrir des pistes didactiques.

\section{Points de vue sur la formation à l'écrit}

Interrogés sur ce qu'ils pensent de leur formation, les trois témoins affirment que celle-ci est « intéressante ». Ils utilisent le verbe «plaire ». La formation peut représenter une opportunité dans leur vie professionnelle. À ce propos, Jean-Louis affirme : «c'est une perche tendue [...] qui est intéressante ». De plus, après sa première journée de formation, Jean-Louis se souvient : «c'était très bien, c'était plus facile que je [ne] l'imaginais. [Au départ], je pensais que c'était très dur. Ils [les formateurs] se sont mis à notre niveau ».

Nous retrouvons cette même notion de mise en confiance dans les propos de Bachir qui considère qu'en mathématiques comme en langue, c'est « plus intéressant » et c'est «plus facile » que ce qu'il faisait à l'école puisque les formatrices sont « du côté des apprenants, chose qui n'existait pas au collège ». Ce qui plait le plus à Bachir, c'est : « réapprendre les bases parce qu'on oublie au fil du temps ce qu'on a appris ». Cette formation permet également de « rafraichir la mémoire » ajoute-t-il. Comme nous le verrons au paragraphe suivant, lorsqu'elle s'exprime à propos de sa formation, Frédérica évoque essentiellement le contenu des séances.

\section{Perception des séances et des contenus}

\section{Le temps : un élément déterminant dans la formation à l'écrit}

À propos de l'organisation de la formation, Jean-Louis affirme : pour « que ça progresse, ce serait bien de faire au moins deux heures par jour [pendant] deux mois, au minimum. Une journée par semaine, ce n'est pas suffisant, on passe souvent du coq à l'âne, on passe d'un sujet à un autre et on ne finit pas toujours ce qu'on commence. [Ce] n'est pas assez intense pour qu'on puisse enregistrer certaines choses, pour mémoriser ».

Ainsi, ces propos mettent en exergue deux notions. Celle du temps (durée et rythme) nécessaire pour apprendre. Cette notion de temps peut être abordée avec les apprenants avant l'entrée en formation. En effet, lorsqu'elle interroge le rapport au savoir des apprenants, Nacira Ait 
Abdesselam (2008) souligne que le temps est un facteur déterminant dans la prise en compte des besoins de formation. Le temps est perçu de manière différenciée. Ce rapport au temps est constitutif du rapport au savoir et donc à la formation, en lien avec le projet de l'apprenant. Trop éloignés dans le temps, les objectifs peuvent devenir inatteignables et donc un facteur de démotivation qui peut entrainer l'abandon de la formation (Dumet, 2009).

La seconde notion relevée dans les propos de Jean-Louis, concerne les modalités de la formation : " la prof ne peut pas s'occuper de tout le monde à la fois, individuellement ». Le nombre de participants (entre 10 et 12 apprenants par séance observée) et le manque de disponibilité de la formatrice représentent pour lui une contrainte pour ses apprentissages. L'hétérogénéité, inter- et intra-individuelle (Leclercq, 1999) dans les compétences à l'écrit qui caractérise les groupes de formation d'adultes suppose donc des apprenants qu'ils mettent en œuvre des stratégies pour une prise en charge autonome de leurs apprentissages.

\section{Les apprenants proposent des pistes d'amélioration}

Dans les différents entretiens que nous avons eus avec les trois témoins, nous leur avons posé une question relative à ce qu'ils pouvaient proposer pour améliorer leur formation ${ }^{3}$. Les réponses concernent avant tout les contenus de formation. Ainsi, à la différence de Jean-Louis et de Frédérica qui souhaiteraient plus de temps consacré à l'étude de la langue, en particulier de la conjugaison et de l'orthographe, Bachir, quant à lui, aimerait ajouter l'histoire aux contenus de sa formation. Il ajoute que cela lui permettrait de «savoir beaucoup de choses » et ainsi de participer à des discussions et de se cultiver.

Dans la mesure où les groupes sont formés de stagiaires venant de cultures et de pays différents, cette demande relative à la culture générale pourrait jouer un rôle dans une imprégnation et une entente interculturelle. Elle pourrait également permettre aux formateurs d'appréhender les représentations culturelles de leurs apprenants, liées à l'apprentissage des compétences langagières. En effet, comme le souligne Hervé Adami (2009), cette notion d'interculturalité peut être abordée comme une démarche didactique, qui permet au formateur d'adopter une posture réflexive relative aux fausses évidences rencontrées dans la situation

3. La question était la suivante : «Si on vous demandait d'ajouter quelque chose dans votre formation, ce serait quoi?» 
d'apprentissage. En d'autres termes, le formateur s'attachera à expliciter (ou faire expliciter) les implicites rencontrés dans les interactions au cours des séances comme par exemple, le vouvoiement, le tutoiement ou d'autres normes socioculturelles.

Concernant les apprentissages langagiers écrits, Frédérica souligne « [qu'] apprendre à écrire, ce n'est pas facile du tout » et souhaiterait faire, comme dans une formation précédente, «plus de dictées ». Cela lui permet de voir ses «progrès ». De même que Frédérica, Jean-Louis aimerait «faire des dictées [...] mais pour ça il faut du temps ». Ces remarques font référence au cadre scolaire et aux habitudes construites dans cet espace social, par les apprenants. D'une part, cela interroge la manière dont la formation d'adultes est présentée aux apprenants à partir de ses spécificités et de ses contraintes, comme la durée et la fréquence des séances, en lien avec le contenu et la façon dont ils sont orchestrés. La présentation de la formation dite « de base » à des adultes peut être réalisée à partir de la mise en relation de leurs besoins, de leurs attentes et de leurs propres contraintes ainsi que de celles liées au dispositif. D'autre part, ces remarques soulèvent également un autre questionnement autour de l'orientation dans les dispositifs de formation. Ainsi, lorsqu'elle évoque sa formation précédente, Frédérica mentionne un dispositif mis en place dans le cadre d'une association. D'après cette dernière, le groupe était restreint (cinq participants) et les besoins similaires, ce qui permettait au formateur de proposer les mêmes tâches pour tous. Cela n'est pas le cas pour la formation que nous avons observée. Les apprenants se retrouvent dans le même espace avec des activités individualisées. Frédérica évoque la difficulté à rester concentrée sur ses tâches : «c'est une confusion terrible dans ma tête ». De plus, elle « veut apprendre à lire et à écrire » alors que « les autres savent déjà lire et écrire ». Elle conclut en disant qu'elle ne se serait pas inscrite dans ce module si elle avait eu connaissance du déroulement de la formation. Nous verrons plus loin que malgré ces attentes insatisfaites, relatives aux modalités de la formation, Frédérica mentionne des changements dans sa pratique de l'écrit.

\section{Les effets de la formation : ce que les apprenants font ou comptent faire de leur formation}

Le plaisir et l'intérêt pour la formation, évoqués au paragraphe précédent, peuvent être interprétés par le fait que les apprenants reconnaissent qu'il y a un changement dans les acquisitions même si cela demeure insuffisant. Soulignons qu'ils disent être plus autonomes dans la gestion 
de leurs documents administratifs même si la hantise de l'orthographe est toujours présente dans leurs propos.

Parmi les trois témoins interrogés, Bachir se trouve dans une dynamique professionnelle et familiale au moment de sa formation. Professionnelle, parce qu'il met à exécution son projet : créer son activité de restauration rapide. Ce projet le conduit à rédiger un questionnaire avec utilisation de l'outil informatique. La rédaction du questionnaire donne lieu à un travail spécifique sur l'écrit. De plus, concernant le cadre familial, Bachir déclare qu'il transmet à ses enfants des nouveaux savoirs en mathématiques et en lecture. Ce transfert des nouveaux savoirs pourrait être accompagné d'un transfert des savoir-faire. En effet, l'explicitation de la dimension métacognitive au cours des séances pourrait favoriser le transfert des savoir-faire du contexte de la formation à celui de la famille.

Malgré l'écart existant entre ses attentes et la réalité, Frédérica mentionne des changements de son usage de l'écrit. Elle constate qu'elle écrit davantage en français, en particulier dans le cadre personnel. Toutefois, Frédérica pense que la formation l'aide surtout « à parler et à être en contact avec les gens ». Ainsi, lorsqu'elle évoque les exercices de mathématiques, à partir d'une fiche d'activités qui porte sur l'usage des pièces de monnaie, Frédérica pense que cela l'aide davantage à s'exprimer à l'oral qu'à améliorer ses compétences à l'écrit. Son principal objectif de formation, qui est d'améliorer l'usage de l'écrit pour aider son fils dans sa scolarité et pour effectuer ses démarches administratives de manière autonome, est donc partiellement atteint.

De même que Frédérica, lorsque l'on évoque avec Jean-Louis ses nouveaux savoirs et savoir-faire éventuellement développés, ce dernier constate que les activités réalisées durant la formation l'aident davantage « dans la vie quotidienne » que dans son travail. Il parle essentiellement de l'orthographe. Lorsqu'il utilise l'ordinateur durant sa formation, il évoque l'orthographe comme une contrainte pour utiliser les moteurs de recherche et écrire les mots clés. Lorsque nous l'interrogeons à la fin de sa formation, Jean-Louis conclut à propos de sa formation : «j' ai vu là où il faut que je travaille, les lacunes en français ».

\section{Conclusion : des représentations à l'auto-évaluation des apprentissages, pistes didactiques}

L'analyse de ces résultats nous conduit à mettre en évidence des points de convergence pour les trois témoins ayant participé à notre enquête. Ces éléments concernent d'une part, des constats relatifs aux attentes, 
aux ressentis et à l'évolution des pratiques liées à l'usage de l'écrit. Les convergences se rapportent d'autre part, aux modalités de la formation et aux demandes d'amélioration.

Parmi les constats, le premier point de convergence concerne les attentes relatives à la formation. En effet, ce qui a conduit les témoins à s'inscrire dans cette formation, c'est le souhait d'améliorer leurs compétences langagières, en particulier scripturales, en vue d'un usage social, professionnel et familial de l'écrit. L'exemple de Bachir montre que les attentes peuvent émerger en cours de formation. Ainsi, les réinterroger, permettrait aux apprenants de les exprimer et aux formateurs d'adapter les contenus au plus près des attentes, en fonction du contexte.

Le second élément de convergence est relatif à une approche psychoaffective de la formation. Pour ces apprenants qui se trouvent, rappelons-le, dans une situation d'insertion professionnelle, cette formation représente une opportunité pour faciliter leur retour à l'emploi. Ils apprécient le climat convivial et le rapport de confiance qui s'établit entre eux et les formateurs. En cela, ils soulignent qu'elle se distingue de ce qu'ils ont vécu dans le cadre scolaire.

Le troisième point de convergence relatif aux constats, concerne les changements évoqués par les témoins à la fin de leur formation. Il s'agit de la transmission de certains contenus (surtout des savoirs) dans le cadre familial. Le transfert des nouveaux savoirs mentionnés se traduit pour certains (Frédérica) par davantage d'aisance dans l'usage de l'écrit, même si ce n'est pas suffisant au regard de leurs attentes.

Les modalités du dispositif de formation sont évoquées à partir d'éléments tels que le temps nécessaire entre deux séances pour la mémorisation des nouveaux savoirs ainsi que l'organisation des séances : difficulté de concentration liée aux bavardages et manque de disponibilité du formateur pour les interventions individuelles. À propos d'éventuelles pistes d'amélioration de leur formation, s'ils mentionnent des idées focalisées sur la micro-syntaxe (conjugaison, orthographe), les témoins proposent également d'élargir les modules à l'histoire, dans le but d'améliorer la culture générale. De plus, la dictée est évoquée comme un exercice permettant de mesurer leur progression dans l'apprentissage de l'écrit. Nous voyons à travers ces remarques que les propos des témoins ne concernent plus seulement les tâches et le contenu des séances mais aussi l'évaluation des acquis et donc le processus d'apprentissage.

Ces constats et remarques mériteraient d'être approfondis en interrogeant un échantillon plus important d'apprenants, dans des organismes 
de formation différents. Toutefois, dans le prolongement de notre recherche, quelques pistes didactiques peuvent être formulées. En effet, les entretiens ont permis de mettre à jour des représentations de la formation relatives aux activités, aux contenus et au dispositif. Dans un premier temps, nous avons recueilli des opinions; puis, un changement s'opère au fil des échanges puisque les témoins apportent des éléments que l'on pourrait considérer comme relevant d'une auto-évaluation de leur formation. Ces propos se caractérisent par une référence au cadre scolaire et par des propositions d'amélioration. Nous assistons alors à un changement de posture qui ne consiste plus seulement à formuler des constats sur les objets d'apprentissage mais à adopter une attitude réflexive vis à vis de la formation, que l'on pourrait aussi qualifier d'évaluative.

Ces situations d'entretien permettent donc de s'interroger sur la notion d'auto-évaluation, en lien avec les représentations et dans ce prolongement, sur la place accordée aux apprenants dans la réflexion menée pour améliorer les dispositifs.

\section{RÉFÉRENCES BIBLIOGRAPHIQUES}

ADAMI H. (2009) : La formation linguistique des migrants, Paris, CLE International.

Ait Abdesselam N. (2008) : « Rapports aux savoirs et perspectives temporelles d'adultes en situation d'illettrisme », Transformations. Recherches en éducation des adultes, $\mathrm{n}^{\circ} 1, \mathrm{p} .81-91$.

BarRé-DE MiniaC C. (2000) : Le rapport à l'écriture. Aspects théoriques et didactiques, Lille, Presses universitaires du Septentrion.

Beillerot J. (1982) : La société pédagogique, Paris, Presses universitaires de France.

Besse J.-M., Petiot-Poirson K. et Petit Charles E. (2003) : Qui est illettré? Décrire et évaluer les difficultés à se servir de l'écrit, Paris, Retz.

Blanchet A. (2005) : "Interviewer», dans A. Blanchet, R. Ghiglione, $\mathrm{J}$. Massonnat et A. Trognon, Les techniques d'enquête en sciences sociales, Paris, Dunod, p. 81-126.

DABÈnE M. (1992) : «Compétences scripturales et pratiques d'écriture », dans J.-M. Besse, M.-M. de Gaulmyn, D. Ginet et B. Lahire (dir.), L'illettrisme en question, Lyon, Presses universitaires de Lyon, p. 101-107.

Dumazedier J. (1978) : « La société éducative et ses incertitudes », Éducation Permanente, $\mathrm{n}^{\circ}$ 44, p. 3-13.

DuMEt T. (2009) : Illettrisme et formation de base. L'épreuve des contradictions, thèse de doctorat en Sciences de l'éducation, université de Lille. 
GRLI (2003) : Une démarche d'évaluation des apprentissages en AFB, Rouen, Centre Ressources Illettrisme Haute-Normandie.

JAFFRÉ J.-P. (2004) : « La litéracie : histoire d'un mot, effets d'un concept », dans C. Barré-de Miniac, C. Brissaud, et M. Rispail (dir.), La littéracie, conceptions théoriques et pratiques d'enseignement de la lectureécriture, Paris, L'Harmattan, p. 21-41.

LECLERCQ V. (1999) : Face à l'illettrisme, enseigner l'écrit à des adultes, Paris, ESF.

Penloup M.-C. (1995) : Le rapport à l'écriture littéraire du scripteur ordinaire, thèse de doctorat des Sciences du langage, Rouen, université de Rouen.

VERMERSCH P. (2006) : L'entretien d'explicitation, Issy-les-Moulinaux, ESF.

VINÉRIER A. (2004) : Des chemins de savoir dans une pédagogie de la conscientisation, Guide pour le formateur, CRDP Orléans-Tours, Imprimerie Nouvelle. 